\section{Acute isolated sphenoid sinusitis in a 4-year-old child: a rare case with an atypical presentation}

AIKATERINI MONTI • MARIA FARINI

Paediatric Department Giannitsa General Hospital Giannitsa, Greece

CONSTANTINE KATSANOULAS $(\bowtie)$ ICU, Hippokrteion General Hospital 85, Mantineias str. 54248 Thessaloniki, Greece Phone: +30 2310892511 +306944595016

E-mail: conkats@the.forthnet.gr

\begin{abstract}
Acute, isolated, sphenoid sinusitis is unusual, especially in children. Its incidence is about $2.7 \%$ of all sinus infections and is even rarer in children under the age of six years. It is frequently misdiagnosed because of its atypical presentation and it can cause serious complications because of the sphenoid sinus' anatomical relations with many intracranial structures. We report a case of a previously healthy 4 year old boy, whose sole symptom at presentation was drowsiness. Physical examination and initial laboratory investigations were normal. Diagnosis was made after computed tomography of the head revealed left sphenoiditis. Although under treatment, the child manifested meningism during the first day of hospitalization, which subsided the next day. He was treated with ceftriaxone plus clindamycin and had a good outcome, without complications or neurological sequelae.
\end{abstract}

Key words: sphenoiditis, intracranial complications, children

\section{Introduction}

The sphenoid sinus is often referred to as the "neglected" sinus. (1) Infection usually occurs in conjunction with infection of the other paranasal sinuses. Isolated acute sphenoiditis is rare (1-3) and it is reported almost uniquely in older children. (4) It merits particular concern, due to potentially serious and even fatal complications. $(2,5-8)$ It is frequently misdiagnosed because of its atypical presentation. $(3,9,10)$

We report a case of a previously healthy 4-year-old boy, presenting with drowsiness.

\section{Case report}

A 4-year-old boy, presented to hospital with somnolence, noticed during the course of the morning despite having slept normally. He had no fever and no signs of infection, nor any history of trauma. His parents declared that he could not reach any medication at home nor had they witnessed any obvious convulsions. His personal and family medical history was insignificant. He was fully immunized.

Initial blood glucose was $8,3 \mathrm{mmol} / \mathrm{L}$. He was somnolent, responding to vocal stimuli with no other neurological signs (pupil size and reactivity, posture, tone, reflexes were normal). No neck stiffness was noted. The remainder of the physical examination was normal.

Differential diagnoses included viral or bacterial CNS infection, brain trauma or cerebro-vascular accident, metabolic or toxicological causes or epileptic post-ictal phase. Lumbar puncture showed clear cerebrospinal fluid (CSF) with 2 white blood cells per microlitre and no other findings. Gram stain and culture was negative. A full blood examination revealed a WBC count of 29000 (85\% neutrophils, 9\% lymphocytes), normal $\mathrm{Hb}$ and platelets. CRP was $0,11 \mathrm{mg} / \mathrm{dl}$ and ESR $8 \mathrm{~mm} / 1 \mathrm{~h}$. All biochemical investigations were within normal range. Urinalysis was clear. Computed tomography (CT) of the head revealed left sphenoid sinus opacity, with the rest of the sinuses and brain (figure 1) normal.

The patient commenced treatment with i.v. ceftriaxone plus clindamycin. Some hours later, he manifested symptoms indicating meningitis (intense headache, photophobia, emesis and neck stiffness). A single dose of methylprednisolone was added. The next day, the meningism subsided, and the rest of his course was uneventful. He was treated with intravenous antibiotics for 10 days, after which he was switched to oral cefuroxime axetil for 10 days. His final outcome was excellent.

\section{Discussion}

The sphenoid sinus has special attributes. It is lined with ciliated pseudostratified epithelium with fewer 


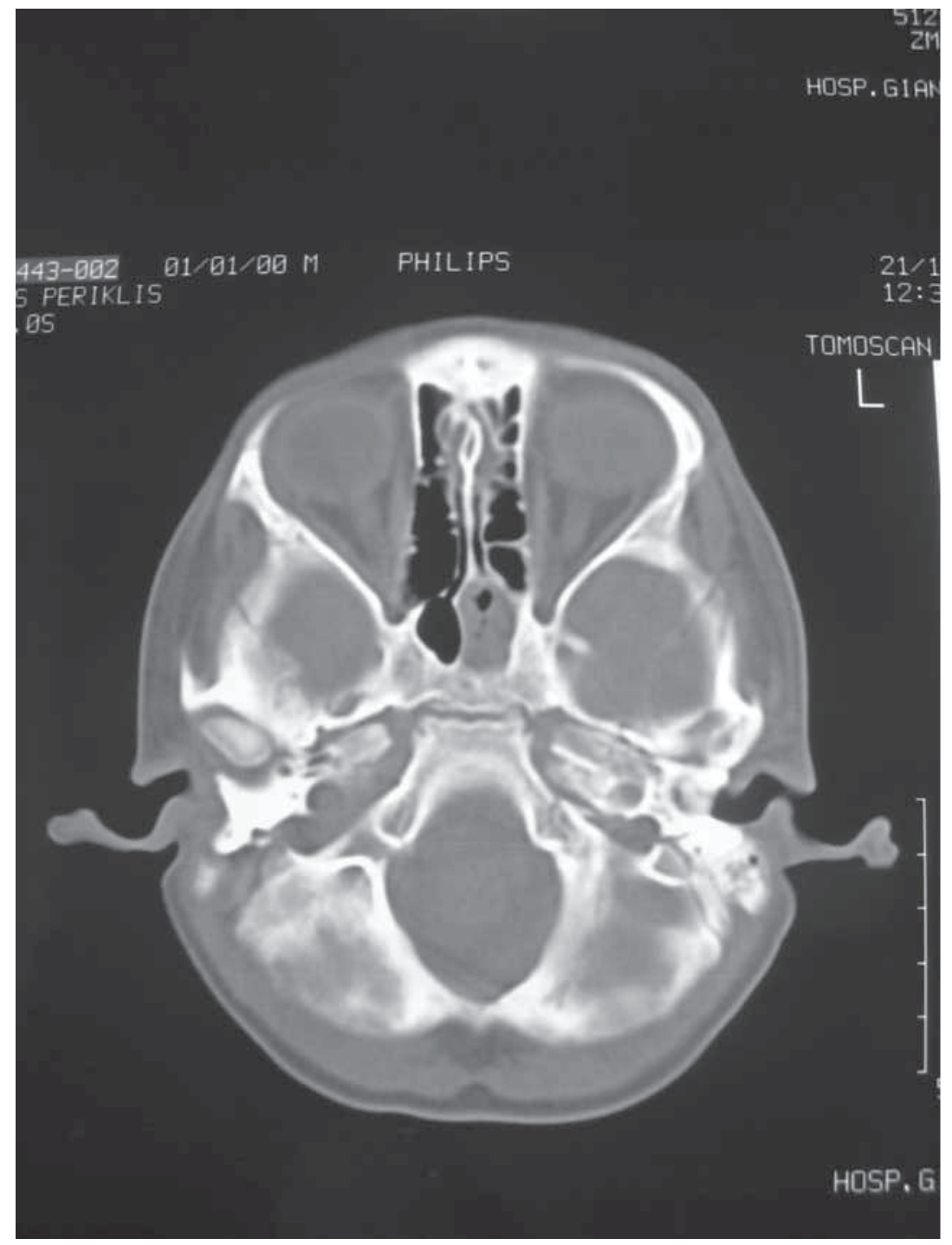

Figure 1. Opacity of the left sphenoid sinus in our patient's head CT.

mucous secreting cells when compared to the other paranasal sinuses. This contributes to fewer drainage problems and may explain the low incidence of isolated sphenoiditis (fewer than $3 \%$ of all sinusitis cases). (3) Most pediatric patients are adolescents and pre-adolescents. Cases in young children are rarer because significant development of the sphenoid sinus does not begin until the age of 4 to 6 years. (4) This was the case of our 4-year-old patient.

Sphenoiditis is of particular interest because the sphenoid sinus' thin wall and special anatomical location facilitate dissemination of infection to many intracranial structures. Approxi- developed symptoms of meningitis, without CSF changes, probably due to direct irritation of the meninges.

Symptoms are usually vague and atypical. $(3,9)$ Headache and visual disturbances are the most common symptoms. $(3,4,8)$ Our patient's sole initial symptom was somnolence, while headache manifested later with other symptoms of meningeal irritation. As in our case, the absence of nasal symptoms does not exclude sphenoiditis. (3) Fever and other signs of infection are usually absent as well. $(4,13)$ Our patient was afebrile and initial laboratory tests did not confirm any bacterial infection.

A $78 \%$ rate of misdiagnosed cases is reported in the literature, attributed to atypical presentation. (10) The diagnostic study of choice is CT scan of the head. Findings may include mucosal thickening, sphenoid opacification, and bony erosions or sclerosis. Bone findings are less usual in sphenoiditis compared to malignancies. Magnetic resonance imaging with i.v. contrast should be obtained in the case of cranial nerve abnormalities. Routine radiographs usually fail to reveal sphenoiditis, because of its deep location. Sphenoid opacity alone on plain X-rays is not diagnostic of sinusitis, except when air/fluid levels are present (figure 2).

In our patient, a CT scan was performed to investigate for intracranial causes, sphenoiditis not being one of our initial diagnoses.

The most common organisms identified in sphenoid sinusitis are S. aureus, S. pneumoniae and other aerobic and anaerobic strains. S. pneumoniae appears to be the pathogen most frequently associated with the complication of meningitis. Fungi, especially Aspergillus sp., should be considered in all patients, particularly if they are immunocompromised. Uncomplicated cases, if diagnosed and treated early with optimal antibiotic therapy, resolve easily. Treatment might include a combination of clindamycin for $S$. aureus, Streptococcus sp. and anaerobes and a third generation cephalosporin for gram-negative organisms, 
given parenterally. Cloxacillin may be added for $\mathrm{S}$. aureus coverage. Patients allergic to cephalosporin can be given aminoglycosides instead. Specific antimicrobial therapy can be prescribed; depending on the results from the CSF, blood or sinus aspirates cultures. Depending on the clinical course, treatment could be continuously parenteral or oral, with total duration of up to 3 or 4 weeks. If symptoms progress or continue for more than 24 hours or if any complication arises, immediate surgical drainage of the sphenoid sinus is indicated, using various available approaches. (3)

In our case, CSF culture was negative and there was no evidence of immunosupression. The treatment was selected empirically, with the most common organisms mentioned above in mind. Our patient received i.v. ceftriaxone plus clindamycin for 10 days followed by 10 days of cefuroxime axetil per os. There was improvement from the second day of treatment and the final outcome was excellent, without any complications or sequelae.

\section{Conclusion}

Acute isolated sphenoid sinusitis is a rare condition, especially in young children. It is usually misdiagnosed because of its atypical manifestations. It may involve several intracranial structures, with potentially severe or even fatal complications. Prompt diagnosis and antibiotic therapy is essential to minimize mortality and morbidity. CT scan of the head is the examination of choice and MRI may be considered, if necessary. Uncomplicated cases can

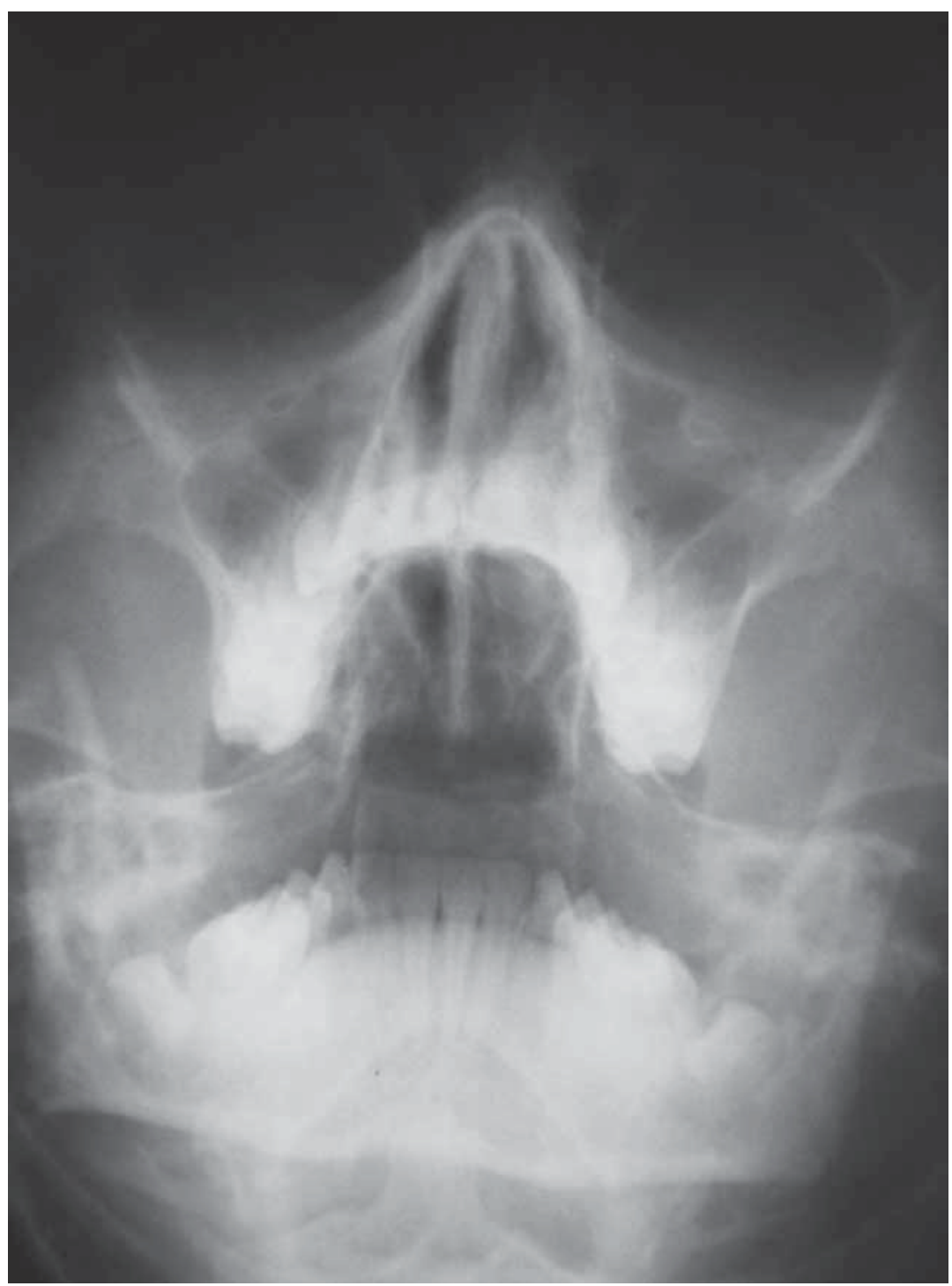

Figure 2. A plain X-ray of the sinuses in an $8 y$ old child: simple opacity of the left sphenoid.

resolve with optimal antibiotic therapy. Persistence or development of intracra- nial complications is an indication for immediate surgical drainage.

\section{REFERENCES}

1. Ng YT, Butler IJ. Sphenoid sinusitis masquerading as migraine headaches in children. J Child Neurol 2001;16:882-4.

2. Uren BA, Berkowitz RG. Isolated inflammatory sphenoid sinus disease in children. Ann Otol Rhinol Laryngol 2003;112:370-2.

3. Tan HHK, Ong YK. Acute isolated sphenoid sinusitis. Ann Acad Med Singap 2004;33:656-9.

4. Haimi-Cohen Y, Amir J, Zeharia A, Danziger Y, Ziv N, Mimouni M. Isolated sphenoid sinusitis in children. Eur J Pediatr 1999;158:298-301.

5. Rimal D, Hashmi SM, Prinsley PR. An unusual presentation of sphenoid sinusitis with septicaemia in a healthy young adult. Emerg Med $\mathrm{J}$ 2006;23:e36.

6. Saitoh A, Beall B, Nizet V. Fulminant bacterial meningitis complicating sphenoid sinusitis. Pediatr Emerg Care 2003;19:415-7. 
7. Radulescu L, Poiata I, Curca IA, Martu D. Thrombophlebitis of left cavernous sinus secondary to acute right sphenoid sinusitis. Rev Med Chir Soc Med Nat lasi 2005;109:573-6.

8. Deans JA, Welch AR. Acute isolated sphenoid sinusitis: a disease with complications. J Laryngol Otol 1991;105:1072-4.

9. Herrmann BW, White FV, Forsen JW. Visual loss in a child due to allergic fungal sinusitis of the sphenoid. Otolaryngol Head Neck Surg 2006;135:328-9.

10. Hnatuk LA, Macdonald RE, Papsin BC. Isolated sphenoid sinusitis: the Toronto Hospital for Sick Children experience and review of the literature. J Otolaryngol 1994;23:36-41.

11. Nemzek W, Postma G, Poirier V, Hecht S. MR features of pachymeningitis presenting with sixth-nerve palsy secondary to sphenoid sinusitis. AJNR Am J Neuroradiol 1995;16:960-3.

12. Ada M, Kaytaz A, Tuskan K, Guvenc MG, Selcuk H. Isolated sphenoid sinusitis presenting with unilateral Vlth nerve palsy. Int J Pediatr Otorhinolaryngol 2004;68:507-10.

13. Goldman GE, Fontanarosa PB, Anderson JM. Isolated sphenoid sinusitis. Am J Emerg Med 1993;11:235-8. 